\title{
The Formation of $\mathrm{H}_{2}$ and $\mathrm{HD}$ with the Master Equation Approach
}

\author{
Ofer Biham ${ }^{1}$, Azi Lipshtat ${ }^{1} \dagger$, and Hagai B. Perets ${ }^{1} \ddagger$ \\ ${ }^{1}$ Racah Institute of Physics, The Hebrew University, Jerusalem 91904, Israel \\ email: biham@phys.huji.ac.il
}

\begin{abstract}
The formation of $\mathrm{H}_{2}$ and $\mathrm{HD}$ molecules on interstellar dust grains is studied using rate equation and master equation models. Rate equations are used in the analysis of laboratory experiments, which examine the formation of molecular hydrogen on astrophysically relevant surfaces. However, under interstellar conditions, rate equations are not suitable for the calculation of reaction rates on dust-grain surfaces. Due to the low flux and the sub-micron size of the grains, the populations of $\mathrm{H}$ and $\mathrm{D}$ atoms on a single grain are likely to be small. In this case the reaction rates are dominated by fluctuations and should be calculated using stochastic methods. The rate of molecular hydrogen formation in interstellar clouds is evaluated using the master equation, taking into account the distribution of grain sizes.
\end{abstract}

Keywords. ISM: abundances — ISM: molecules — molecular processes

\section{Introduction}

The formation of molecular hydrogen in the interstellar medium is a process of fundamental importance in astrophysics. $\mathrm{H}_{2}$ molecules contribute to the cooling of clouds during gravitational collapse and participate in reactions that produce more complex molecules (Duley \& Williams 1984; Williams 1998). $\mathrm{H}_{2}$ cannot form in the gas phase efficiently enough to account for its observed abundance (Gould \& Salpeter 1963). It was thus proposed that $\mathrm{H}_{2}$ formation takes place on dust grains that act as catalysts. The process of $\mathrm{H}_{2}$ formation on grains can be broken up into several steps as follows. An $\mathrm{H}$ atom approaching the surface of a grain has a probability $\xi$ to stick and become adsorbed. The adsorbed $\mathrm{H}$ atom (adatom) resides on the surface for an average time $t_{\mathrm{H}}$ (residence time) before it desorbs. In the Langmuir-Hinshelwood mechanism, the adsorbed $\mathrm{H}$ atoms quickly equilibrate and diffuse on the surface of the grain either by thermal activation or by tunneling. When two adsorbed $\mathrm{H}$ atoms encounter each other, an $\mathrm{H}_{2}$ molecule may form (Williams 1968; Hollenbach \& Salpeter 1970; Hollenbach \& Salpeter 1971; Hollenbach et al. 1971; Smoluchowski 1981; Aronowitz \& Chang 1985; Duley \& Williams 1986; Pirronello \& Averna 1988; Sandford \& Allamandolla 1993; Takahashi et al. 1999; Farebrother et al. 2000). The steady-state production rate of molecular hydrogen, $R_{\mathrm{H}_{2}}$ $\left(\mathrm{cm}^{-3} \mathrm{~s}^{-1}\right)$ can be expressed by

$$
R_{\mathrm{H}_{2}}=\frac{1}{2} n_{\mathrm{H}} v_{\mathrm{H}} \sigma \gamma n_{\mathrm{g}},
$$

where $n_{\mathrm{H}}\left(\mathrm{cm}^{-3}\right)$ and $v_{\mathrm{H}}\left(\mathrm{cm} \mathrm{s}^{-1}\right)$ are the number density and the speed of $\mathrm{H}$ atoms in the gas phase, respectively, $\sigma\left(\mathrm{cm}^{2}\right)$ is the average cross-sectional area of a grain and $n_{\mathrm{g}}\left(\mathrm{cm}^{-3}\right)$ is the number density of dust grains. The parameter $\gamma$ is the fraction of $\mathrm{H}$

$\dagger$ Present address: Department of Pharmacology and Biological Chemistry, Mount Sinai School of Medicine, New York, NY 10029, USA.

$\ddagger$ Present address: Faculty of Physics, Weizmann Institute of Science, Rehovot 76100, Israel. 
atoms striking the grain that eventually form a molecule, namely $\gamma=\xi \eta$, where $\eta$ is the probability that an $\mathrm{H}$ adatom on the surface will recombine with another $\mathrm{H}$ atom to form $\mathrm{H}_{2}$. The probability $\xi$ for an $\mathrm{H}$ atom to become adsorbed on a grain surface covered by an ice mantle was calculated by Buch \& Zhang (1991) and Masuda et al. (1998). They found that $\xi$ depends on the surface temperature and on the energy of the irradiation beam. For a surface at $10 \mathrm{~K}$ and beam temperature of $350 \mathrm{~K}$, Masuda et al. (1998) obtained a sticking coefficient around 0.5.

A simplified version of Eq. (1.1) is often used for the evaluation of the $\mathrm{H}_{2}$ formation rate in models of interstellar chemistry. It takes the form

$$
R_{\mathrm{H}_{2}}=R \cdot n_{\mathrm{H}} n,
$$

where $R\left(\mathrm{~cm}^{3} \mathrm{~s}^{-1}\right)$ is the rate coefficient and $n=n_{\mathrm{H}}+2 n_{\mathrm{H}_{2}}\left(\mathrm{~cm}^{-3}\right)$ is the total density of hydrogen atoms in both atomic and molecular $\left(n_{\mathrm{H}_{2}}\right)$ form. In previous studies it was assumed that the total grain mass is about $1 \%$ of the hydrogen mass in the cloud, with a single grain size of $r=0.17 \mu \mathrm{m}$ and a grain mass density of $\rho_{g}=2\left(\mathrm{~g} \mathrm{~cm}^{-3}\right)$ (Hollenbach et al. 1971). Under these assumptions, for gas temperature of $100 \mathrm{~K}$ and $\gamma=0.3$, one obtains $R \simeq 10^{-17}\left(\mathrm{~cm}^{3} \mathrm{~s}^{-1}\right)$, in agreement with observations for diffuse clouds (Jura 1975). More recent observations in photon dominated regions indicate that in these regions the rate coefficient should be $R \simeq 10^{-16}\left(\mathrm{~cm}^{3} \mathrm{~s}^{-1}\right)$, in order to account for the observed $\mathrm{H}_{2}$ abundance (Habart et al. 2003).

\section{Laboratory Experiments}

In the last few years, the formation of molecular hydrogen on interstellar dust analogues was studied in a series of laboratory experiments (Pirronello et al. 1997a; Pirronello et al. 1997b; Pirronello et al. 1999; Roser et al. 2002; Roser et al. 2003). In these experiments, beams of $\mathrm{H}$ and $\mathrm{D}$ atoms were irradiated on the surface. The production of HD molecules was measured both during irradiation and during a subsequent temperature programmed desorption (TPD) experiment. In this experiment, the temperature of the sample is raised quickly to either desorb particles (atoms and molecules) that got trapped on the surface or to enhance their diffusion and subsequent reaction and desorption. The formation of molecular hydrogen was studied on samples of polycrystalline olivine (Pirronello et al. 1997a; Pirronello et al. 1997b), amorphous carbon (Pirronello et al. 1999) and various types of amorphous ice samples, namely high density ice (HDI) and low density ice (LDI) (Manicó et al. 2001; Roser et al. 2002; Hornekær et al. 2003). The results of the TPD experiments, particularly on polycrystalline olivine and HDI, show second-order kinetics for low coverage of $\mathrm{H}$ and $\mathrm{D}$ atoms on the surface. In second-order kinetics the peak shifts towards higher temperature as the coverage is reduced. This is due to the larger distancees that atoms need to diffuse before they encounter each other and recombine. This indicates that on these samples the surface mobility of $\mathrm{H}$ and $\mathrm{D}$ atoms is dominated by thermal hopping rather than tunneling. If tunneling were dominant, molecules could form efficiently during irradiation, resulting in first-order kinetics.

The results of the TPD experiments were analyzed using rate equation models (Katz et al. 1999; Cazaux \& Tielens 2002; Cazaux \& Tielens 2004; Perets et al. 2005). The analysis involves fitting the parameters of the rate equations to the experimental TPD curves. The most important parameters are the activation energy barriers for diffusion and desorption of hydrogen atoms on the surface. The models used by Katz et al. (1999) and Perets et al. (2005) take into account only physisorption sites and thermal hopping between them. The models of Cazaux \& Tielens $(2002,2004)$ also take into account tunneling and chemisorption sites. However, the two analyses are in agreement on the fact 
that tunneling between physisorption sites appears to be too slow to play an important role in the TPD experiments (Vidali et al. 2005). Furthermore, recent experiments and calculations indicate that there are energy barriers, which are likely to prevent adsorbed $\mathrm{H}$ and $\mathrm{D}$ atoms from entering chemisorption sites at these low temperatures.

Using the parameters obtained in the laboratory experiments, the rate equations enabled the extrapolation from laboratory time scales to astrophysical time scales. Using rate equations, the efficiency of molecular hydrogen formation on these surfaces under interstellar conditions and its dependence on the flux and grain temperature were calculated. It was found that the recombination efficiency strongly depends on the grain temperature. Each sample exhibits a narrow window of high efficiency along the temperature axis. For the polycrystalline olivine sample, this window is between 7-9 K, while for amorphous carbon it is between 11-17 K (Vidali et al. 2005).

\section{The Rate-Equation Model}

Consider a diffuse interstellar cloud with a density $n_{\mathrm{H}}\left(\mathrm{cm}^{-3}\right)$ of $\mathrm{H}$ atoms, and a density $n_{\mathrm{g}}$ of dust grains. The typical velocity $v_{\mathrm{H}}\left(\mathrm{cm} \mathrm{s}^{-1}\right)$ of $\mathrm{H}$ atoms in the gas phase is given by

$$
v_{\mathrm{H}}=\sqrt{\frac{8}{\pi} \frac{k_{B} T_{\mathrm{gas}}}{m_{\mathrm{H}}}},
$$

where $m_{\mathrm{H}}=1.67 \times 10^{-24}$ (gram) is the mass of an $\mathrm{H}$ atom and $T_{\text {gas }}$ is the gas temperature. To evaluate the flux of atoms onto grain surfaces we will assume, for simplicity, that the grains are spherical with a radius $r(\mathrm{~cm})$. The cross section of a grain is $\sigma=\pi r^{2}$ and its surface area is $4 \sigma$. The density of adsorption sites on the surface is $s$ (sites $\mathrm{cm}^{-2}$ ) and the number of adsorption sites on a grain is $S=4 \pi r^{2} s$. The flux $F_{\mathrm{H}}$ (atoms s${ }^{-1}$ ) of $\mathrm{H}$ atoms onto the surface of a single grain is given by $F_{\mathrm{H}}=n_{\mathrm{H}} v_{\mathrm{H}} \sigma$.

$\mathrm{H}$ atoms that collide and stick to the surface hop as random walkers between adjacent sites until they either desorb or recombine into molecules. The desorption rate of an $\mathrm{H}$ atom is

$$
W_{\mathrm{H}}=\nu \cdot \exp \left(-E_{1} / k_{B} T\right),
$$

where $\nu$ is the attempt rate (typically assumed to be $10^{12} \mathrm{~s}^{-1}$ ), $E_{1}$ is the activation energy barrier for desorption of an $\mathrm{H}$ atom and $T$ is the surface temperature. The hopping rate of an $\mathrm{H}$ atom is

$$
a_{\mathrm{H}}=\nu \cdot \exp \left(-E_{0} / k_{B} T\right),
$$

where $E_{0}$ is the activation energy barrier for $\mathrm{H}$ diffusion. Throughout this paper we use the parameters obtained experimentally for amorphous carbon, namely the activation energies $E_{0}=44.0 \mathrm{meV}$ and $E_{1}=56.7 \mathrm{meV}$ (Katz et al. 1999), and the density of adsorption sites on the surface $s \simeq 5 \times 10^{13}\left(\right.$ sites $\left.\mathrm{cm}^{-2}\right)$ (Biham et al. 2001). For the density of hydrogen atoms in the gas phase we take $n_{\mathrm{H}}=10$ (atoms $\left.\mathrm{cm}^{-3}\right)$. The gas temperature is taken as $T_{\text {gas }}=90 \mathrm{~K}$, thus $v_{\mathrm{H}}=1.37 \times 10^{5}\left(\mathrm{~cm} \mathrm{~s}^{-1}\right)$. These are typical values for diffuse interstellar clouds.

The number of $\mathrm{H}$ atoms on the grain is denoted by $N_{\mathrm{H}}$ and its expectation value is denoted by $\left\langle N_{\mathrm{H}}\right\rangle$. The rate $A_{\mathrm{H}}=a_{\mathrm{H}} / S$ is approximately the inverse of the time $t_{s}$ required for an atom to visit nearly all the adsorption sites on the grain surface. This is due to the fact that in two dimensions the number of distinct sites visited by a random walker is linearly proportional to the number of steps, up to a logarithmic correction (Montroll \& Weiss 1965). 
The formation of $\mathrm{H}_{2}$ molecules on interstellar dust grains can be described by the rate equation

$$
\frac{d\left\langle N_{\mathrm{H}}\right\rangle}{d t}=F_{\mathrm{H}}-W_{\mathrm{H}}\left\langle N_{\mathrm{H}}\right\rangle-2 A_{\mathrm{H}}\left\langle N_{\mathrm{H}}\right\rangle^{2} .
$$

The first term on the right-hand side describes the flux of $\mathrm{H}$ atoms, the second term describes the desorption of $\mathrm{H}$ atoms and the third term describes the diffusion and recombination. Here we assume, for simplicity, that all $\mathrm{H}_{2}$ molecules desorb from the surface upon formation. The production rate $R_{\mathrm{H}_{2}}\left(\mathrm{~cm}^{-3} \mathrm{~s}^{-1}\right)$ of $\mathrm{H}_{2}$ molecules is given by

$$
R_{\mathrm{H}_{2}}=A_{\mathrm{H}}\left\langle N_{\mathrm{H}}\right\rangle^{2} n_{\mathrm{g}} .
$$

By solving the rate equations under steady-state conditions for astrophysically relevant values of the flux and temperature, it was found (Biham \& Lipshtat 2002) that molecular hydrogen formation is efficient only within a narrow window of grain temperatures, $T_{0}<$ $T<T_{1}$, where

$$
T_{0}=\frac{E_{0}}{k_{\mathrm{B}} \ln \left(\nu S / F_{\mathrm{H}}\right)}
$$

and

$$
T_{1}=\frac{2 E_{1}-E_{0}}{k_{\mathrm{B}} \ln \left(\nu S / F_{\mathrm{H}}\right)} .
$$

At temperatures below $T_{0}$, the mobility of $\mathrm{H}$ atoms on the surface is very low, sharply reducing the production rate. At temperatures above $T_{1}$ most atoms quickly desorb before they encounter each other and form molecules.

Rate equations are an ideal tool for the simulation of surface reactions, due to their simplicity and high computational efficiency. In particular, they account correctly for the temperature dependence of the reaction rates. However, in the limit of small grains under low flux they become unsuitable. This is because they ignore the fluctuations as well as the discrete nature of the populations of atoms on the grain (Charnley et al. 1997; Caselli et al. 1998; Shalabiea et al. 1998; Stantcheva et al. 2001). For example, as the number of $\mathrm{H}$ atoms on a grain fluctuates in the range of 0,1 or 2 , the $\mathrm{H}_{2}$ formation rate cannot be obtained from the average number alone. This can be easily understood, since the recombination process requires at least two $\mathrm{H}$ atoms simultaneously on the surface. In this limit, the master equation approach is needed in order to evaluate the $\mathrm{H}_{2}$ formation rate.

\section{The Master Equation}

Consider a grain exposed to a flux of $\mathrm{H}$ atoms. The probability that there are $N_{\mathrm{H}}$ hydrogen atoms on its surface is given by $P_{\mathrm{H}}\left(N_{\mathrm{H}}\right)$, where $N_{\mathrm{H}}=0,1,2, \ldots, S$. The master equation provides the time derivatives of these probabilities. It is a set of coupled linear differential equations of the form

$$
\begin{aligned}
\dot{P}_{\mathrm{H}}\left(N_{\mathrm{H}}\right)= & F_{\mathrm{H}}\left[P_{\mathrm{H}}\left(N_{\mathrm{H}}-1\right)-P_{\mathrm{H}}\left(N_{\mathrm{H}}\right)\right]+W_{\mathrm{H}}\left[\left(N_{\mathrm{H}}+1\right) P_{\mathrm{H}}\left(N_{\mathrm{H}}+1\right)-N_{\mathrm{H}} P_{\mathrm{H}}\left(N_{\mathrm{H}}\right)\right] \\
& +A_{\mathrm{H}}\left[\left(N_{\mathrm{H}}+2\right)\left(N_{\mathrm{H}}+1\right) P_{\mathrm{H}}\left(N_{\mathrm{H}}+2\right)-N_{\mathrm{H}}\left(N_{\mathrm{H}}-1\right) P_{\mathrm{H}}\left(N_{\mathrm{H}}\right)\right] .
\end{aligned}
$$

Each equation includes three terms. The first term describes the effect of the incoming flux $F_{\mathrm{H}}$. The probability $P_{\mathrm{H}}\left(N_{\mathrm{H}}\right)$ increases when an $\mathrm{H}$ atom is adsorbed on a grain that already has $N_{\mathrm{H}}-1$ adsorbed atoms [at a rate of $F_{\mathrm{H}} P_{\mathrm{H}}\left(N_{\mathrm{H}}-1\right)$ ], and decreases when a new atom is adsorbed on a grain with $N_{\mathrm{H}}$ atoms on it [at a rate of $F_{\mathrm{H}} P_{\mathrm{H}}\left(N_{\mathrm{H}}\right)$ ]. The second term includes the effect of desorption. An $\mathrm{H}$ atom that is desorbed from a grain with $N_{\mathrm{H}}$ atoms decreases the probability $P_{\mathrm{H}}\left(N_{\mathrm{H}}\right)$ [at a rate of $N_{\mathrm{H}} W_{\mathrm{H}} P_{\mathrm{H}}\left(N_{\mathrm{H}}\right)$ ], and increases 
the probability $P_{\mathrm{H}}\left(N_{\mathrm{H}}-1\right)$ at the same rate. The third term describes the effect of $\mathrm{H}_{2}$ formation. The production of one molecule reduces the number of $\mathrm{H}$ atoms on the surface from $N_{\mathrm{H}}$ to $N_{\mathrm{H}}-2$. For one pair of $\mathrm{H}$ atoms the recombination rate is proportional to the sweeping rate $A_{\mathrm{H}}$ multiplied by 2 , since both atoms are mobile simultaneously. This rate is multiplied by the number of possible pairs of atoms, namely $N_{\mathrm{H}}\left(N_{\mathrm{H}}-1\right) / 2$. Note that the equations for $\dot{P}_{\mathrm{H}}(0)$ and $\dot{P}_{\mathrm{H}}(1)$ do not include all the terms, because at least one $\mathrm{H}$ atom is required for desorption to occur and at least two for recombination. The rate of formation of $\mathrm{H}_{2}$ molecules on a single grain, $R_{\mathrm{H}_{2}}^{\text {grain }}\left(\mathrm{cm}^{-3} \mathrm{~s}^{-1}\right)$, can be obtained from summation of master equation terms to yield

$$
R_{\mathrm{H}_{2}}^{\text {grain }}=A_{\mathrm{H}}\left[\left\langle N_{\mathrm{H}}^{2}\right\rangle-\left\langle N_{\mathrm{H}}\right\rangle\right]
$$

where

$$
\left\langle N_{\mathrm{H}}^{k}\right\rangle=\sum_{N_{\mathrm{H}}=0}^{\infty} N_{\mathrm{H}}^{k} P_{\mathrm{H}}\left(N_{\mathrm{H}}\right) .
$$

is the $k$-th moment of the distribution $P_{\mathrm{H}}\left(N_{\mathrm{H}}\right)$.

The time dependence of $R_{\mathrm{H}_{2}}^{\text {grain }}$ can be obtained by numerically integrating Eqs. (4.1) using a standard Runge-Kutta stepper. For the case of steady state, namely $\dot{P}_{\mathrm{H}}\left(N_{\mathrm{H}}\right)=0$ for all $N_{\mathrm{H}}$, an analytical solution for $P_{\mathrm{H}}\left(N_{\mathrm{H}}\right)$ was obtained, in terms of $A_{\mathrm{H}} / W_{\mathrm{H}}$ and $W_{\mathrm{H}} / F_{\mathrm{H}}$ (Green et al. 2001; Biham \& Lipshtat 2002). The rate of formation of $\mathrm{H}_{2}$ molecules on a single grain with $S$ adsorption sites is given by (Green et al. 2001; Biham \& Lipshtat 2002)

$$
R_{\mathrm{H}_{2}}^{\text {grain }}=\frac{F_{\mathrm{H}}}{2} \frac{I_{W_{\mathrm{H}} / A_{\mathrm{H}}+1}\left(2 \sqrt{2 F_{\mathrm{H}} / A_{\mathrm{H}}}\right)}{I_{W_{\mathrm{H}} / A_{\mathrm{H}}-1}\left(2 \sqrt{2 F_{\mathrm{H}} / A_{\mathrm{H}}}\right)},
$$

where $I_{x}(y)$ is the modified Bessel function.

In Figure 1, we present the $\mathrm{H}_{2}$ production rate on a single grain of amorphous carbon vs. the grain size, which is parameterized by the number of adsorption sites, $S$. The conditions, specified in $\S 3$ are typical in diffuse clouds and the grain temperature is $T=18 \mathrm{~K}$. It is observed that for large grains the rate equation results coincide with those of the master equation. However, for small grains the rate equation over-estimates the production. The master equation accounts correctly for the rate of $\mathrm{H}_{2}$ formation on grains of all sizes.

\section{Effects of the Grain Size Distribution}

Interstellar dust grains exhibit a broad size distribution between several nanometers and fractions of a micron. This distribution can be approximated by a power law of the form (Mathis et al. 1977; Weingartner \& Draine 2001)

$$
n_{g}(r)=c r^{-\alpha}
$$

where $n_{g}(r) d r\left(\mathrm{~cm}^{-3}\right)$ is the density of grains with radii in the range $(r, r+d r)$ in the cloud. This distribution is bounded between the upper cutoff $r_{\max }=0.25 \mu \mathrm{m}$ and the lower cutoff $r_{\min }=5 \mathrm{~nm}$. The exponent is $\alpha \simeq 3.5$ (Draine \& Lee 1984). Clearly, this distribution is dominated by the small grains. This fact has important implications on the formation rate of molecular hydrogen. The total surface area is much larger than in the case of a homogeneous population of grains with radii of the order of $0.25 \mu \mathrm{m}$, with the same total mass density of about $1 \%$ of the hydrogen mass density. This tends to enhance the $\mathrm{H}_{2}$ formation rate. However, below some grain size the efficiency of $\mathrm{H}_{2}$ 


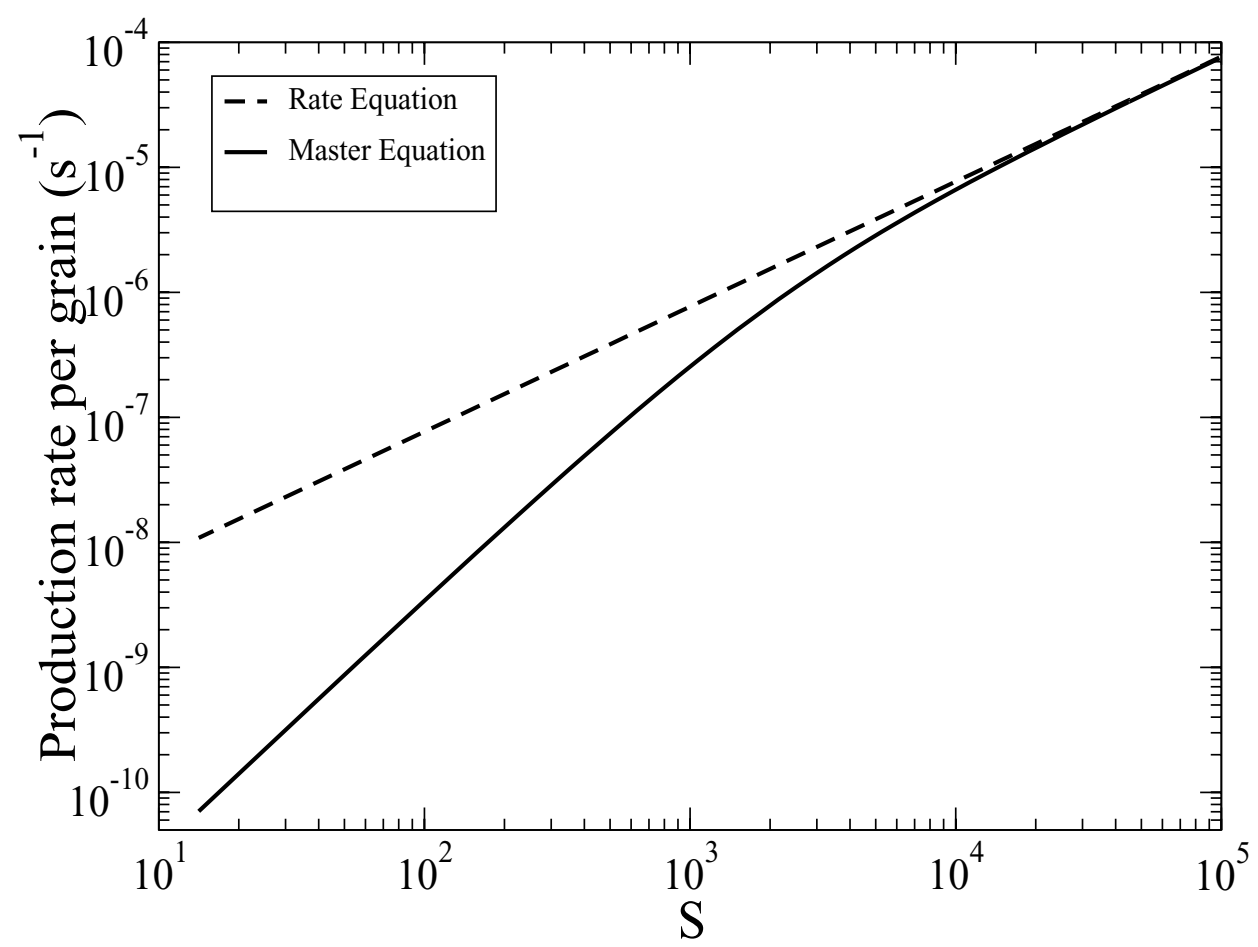

Figure 1. The production rate of $\mathrm{H}_{2}$ molecules on a single grain of amorphous carbon with $S$ adsorption sites. For large grains the rate equation results (dashed line) are in agreement with the master equation (solid line), while for small grains the rate equation over-estimates the production. The parameters are specified in $\S 3$.

formation is sharply reduced. Due to these competing effects the production of molecular hydrogen should be integrated over the whole range of grain sizes.

In Figure 2 we present the rate coefficient $R\left(\mathrm{~cm}^{3} \mathrm{~s}^{-1}\right)$ vs. grain temperature for the case of a power-law distribution of grain sizes (solid line) and for the case, in which all grains are of radius $r=0.17 \mu \mathrm{m}$, with the same total mass of grains. It turns out that, within the temperature window in which $\mathrm{H}_{2}$ formation is efficient, the increase in the total surface area due to the broad size distribution, enhances the production rate by about an order of magnitude compared to a homogeneous population of sub-micron-size grains (Lipshtat \& Biham 2005). This is in spite of the reduced efficiency of $\mathrm{H}_{2}$ formation on small grains.

\section{Effects of Grain Porosity}

The laboratory experiments on interstellar dust analogues have shown that $\mathrm{H}_{2}$ formation on dust grains is efficient within a range of grain temperatures below $20 \mathrm{~K}$. However, high abundances of $\mathrm{H}_{2}$ have also been observed in warmer clouds, including photon-dominated regions (PDRs), where grain temperatures may reach $50 \mathrm{~K}$. The possible effect of grain porosity on $\mathrm{H}_{2}$ formation was recently examined using a rate equation model (Perets \& Biham 2005). It is found that porosity extends the temperature range in which $\mathrm{H}_{2}$ formation is efficient towards higher temperatures. This is because $\mathrm{H}$ atoms that desorb from the internal surfaces of the pores may re-adsorb many times and thus stay longer on the surface. However, this extension is expected to be of only several 


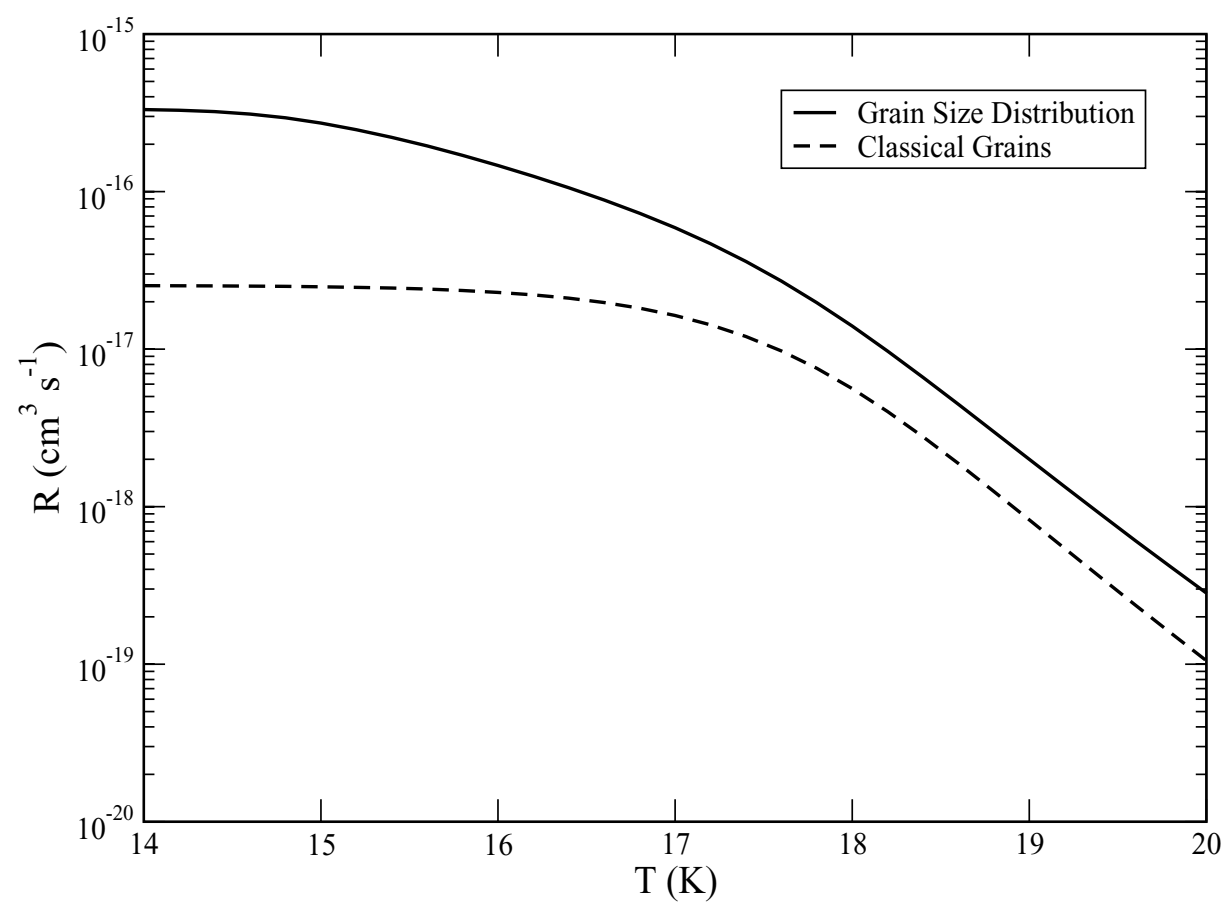

Figure 2. The rate coefficient $R\left(\mathrm{~cm}^{3} \mathrm{~s}^{-1}\right)$ of $\mathrm{H}_{2}$ formation vs. grain temperature, obtained from the master equation for the case of a power-law distribution of grain sizes (solid line). The dashed line shows the rate coefficient for the case in which the same mass of grains consists of a homogeneous population of "classical grains" of radius $r=0.17 \mu \mathrm{m}$.

degrees $(\mathrm{K})$ and by itself cannot account for the formation of molecular hydrogen in PDRs.

Another effect of porosity that may be relevant to molecular hydrogen formation is that porous grains may be significantly colder than predicted by models which use compact, spherical grains (Blanco \& Bussoletti 1980; Voshchinnikov et al. 1999). While models of spherical grains predict grain temperatures in the range of $30-50 \mathrm{~K}$ in PDRs, the actual temperatures of porous interstellar grains may thus be significantly lower. In the most favorable cases, the grain temperatures may go down to the range of $15-30 \mathrm{~K}$ for large grains and to $10-20 \mathrm{~K}$ for small grains (Blanco \& Bussoletti 1980).

\section{HD Formation on Grains}

Unlike the $\mathrm{H}_{2}$ molecules in the interstellar medium which do not form efficiently in the gas phase, HD molecules have an efficient gas-phase formation mechanism. It consists of a sequence of reactions in which an ionized $\mathrm{D}$ atom reacts with an $\mathrm{H}_{2}$ molecule to form HD. The possibility that in diffuse clouds some HD molecules are also formed on grain surfaces was recently examined (Lipshtat et al. 2004). Due to the lower abundance of $\mathrm{D}$ atoms vs. $\mathrm{H}$ atoms (by a factor of about $10^{-5}$ ), the master equation is required for such calculations even on relatively large grains. In case the D atoms are bound more strongly to the surface than $\mathrm{H}$ atoms, the temperature window in which HD formation is efficient is extended towards higher temperatures, compared to the range of efficient $\mathrm{H}_{2}$ formation. The assumption that $\mathrm{D}$ atoms are more strongly bound to the grains is plausible, but so far has not been confirmed experimentally. 


\section{Complex Reaction Networks of Multiple Species}

The master equation can be generalized to describe complex reaction networks of multiple species on grain surfaces. Recently, it was applied to the study of the reaction network that lead to the formation of $\mathrm{H}_{2} \mathrm{CO}$ and $\mathrm{CH}_{3} \mathrm{OH}$ on grains (Stantcheva et al. 2002; Stantcheva \& Herbst 2003). Such surface reaction networks are dominated by $\mathrm{H}$ atoms because hydrogen is the most abundant specie and also because it reacts with most of the other atomic and molecular species adsorbed on grain surfaces. Furthermore, hydrogen atoms hop on the surface much faster than other adsorbed species and thus quickly react with other species even at very low coverages. For such reaction networks, the dynamical variables in the master equation are the probabilities $P\left(N_{\mathrm{H}}, N_{\mathrm{O}}, N_{\mathrm{OH}}, \ldots\right)$ where there are $N_{\mathrm{X}}$ copies of specie X on the grain. Setting suitable cutoffs, each population of each specie is limited to $N_{\mathrm{X}}=0,1, \ldots, N_{\mathrm{X}}^{\max }$. The problem is that as the number of reactive species increases, the number of coupled equations in the master equation increases exponentially, limiting the feasibility of this approach. Using the fact that the reaction networks are usually sparse, it is possible to dramatically reduce the number of equations (Lipshtat \& Biham 2004). This makes the master equation applicable even for complex surface reaction networks with a large number of reactive species.

The master equation can be solved either by direct integration, as described above, or by Monte Carlo (MC) methods (Charnley 2001). A significant advantage of direct integration is that the equations can be easily coupled to the rate equations of gas-phase chemistry. Furthermore, a single run of the direct integration method provides the entire probability distribution of populations of adsorbed species for the simulated conditions as well as the reaction rates. This is in contrast with the MC methods, which typically require large computational resources due to the need to accumulate much statistical information.

\section{Summary}

We have presented the master equation approach for the evaluation of molecular hydrogen formation rates on interstellar dust grains. This approach is required in the limit of small grains and low flux where the population of hydrogen atoms on each grain is small. In this limit, the reaction rate is dominated by fluctuations. Thus, the rate equation approach, which incorporates the mean-field approximation, fails. Applications of the master equation approach for the evaluation of the formation rates of $\mathrm{H}_{2}$, $\mathrm{HD}$ as well as more complex molecules are discussed.

\section{Acknowledgements}

We thank E. Herbst, V. Pirronello and G. Vidali for helpful discussions. This work was supported by the Israel Science Foundation and the Adler Foundation for Space Research.

\section{References}

Aronowitz, S. \& Chang, S. 1985, Ap. J. 293, 243

Biham, O., Furman, I., Pirronello, V., \& Vidali, G. 2001, Ap. J. 553, 595.

Biham, O. \& Lipshtat A. 2002, Phys. Rev. E 66, 055103

Blanco, A. \& Bussoletti, B. 1980, Ap. Space Sci. 67, 105

Buch, V. \& Zhang, Q. 1991, Ap. J. 379, 647

Cazaux, S. \& Tielens, A.G.G.M. 2002, Ap. J. 575, L29

Cazaux, S. \& Tielens, A.G.G.M. 2004, Ap. J. 604, 222 
Charnley, S.B., Tielens, A.G.G.M., \& Rodgers, S.D. 1997, Ap. J. 482, L203

Charnley, S.B. 2001, Ap. J. 562, L99

Caselli, P., Hasegawa, T.I., \& Herbst, E. 1998, Ap. J. 495, 309

Draine B.T. \& Lee, H.M. 1984, Ap. J. 285, 89

Duley, W.W. \& Williams, D.A. 1984, Interstellar Chemistry (Academic Press, London)

Duley, W.W. \& Williams, D.A. 1986, MNRAS 223, 177

Farebrother, A.J., Meijer, A.J.H.M., Clary, D.C., \& Fisher, A.J. 2000, Chem. Phys. Lett. 319, 303

Gould, R.J. \& Salpeter, E.E. 1963, Ap. J. 138, 393

Green, N.J.B., Toniazzo, T., Pilling, M.J. Ruffle, D.P. Bell, N., \& Hartquist, T.W. 2001, A\& A 375,1111

Habart, E., Boulanger, F., Verstraete, L., Pineau des Forêts, G., Falgarone, E., \& Abergel, A. 2003, A\&A 397, 623

Hollenbach, D. \& Salpeter, E.E. 1970, J. Chem. Phys. 53, 79

Hollenbach, D. \& Salpeter, E.E. 1971, Ap. J. 163, 155

Hollenbach, D., Werner M.W., \& Salpeter, E.E. 1971, Ap. J. 163, 165

Hornekær, L., Baurichter, A., Petrunin, V.V., Field, D., \& Luntz, A.C. 2003, Science 302, 1943 Jura, M. 1975, Ap. J. 197, 575

Katz, N., Furman, I., Biham, O., Pirronello V., \& Vidali, G. 1999, Ap. J. 522, 305

Lipshtat, A. \& Biham, O. 2004, Phys. Rev. Lett. 93, 170601

Lipshtat, A., Biham, O., \& Herbst E., 2004, MNRAS 348, 1055

Lipshtat, A. \& Biham, O. 2005, MNRAS 362, 666

Manicó, G., Raguni, G., Pirronello, V., Roser, J.E., \& Vidali G. 2001, Ap. J. 548, L253

Masuda, K., Takahashi, J., \& Mukai, T. 1998, A\& A 330, 773

Mathis, J.S., Rumpl, W., \& Nordsieck, K.H. 1977, Ap. J. 217, 425

Montroll, E.W. \& Weiss, G.H. 1965, J. Math. Phys. 6, 167

Perets, H.B., Biham, O., Manicó, G., Pirronello, V., Roser, J., Swords, S., \& Vidali, G. 2005, Ap. J. 627, 850

Perets, H.B. \& Biham, O. 2005, preprint

Pirronello, V. \& Averna D. 1988, A\&A 201, 196

Pirronello, V., Liu, C., Shen L., \& Vidali, G. 1997a, Ap. J. 475, L69

Pirronello, V., Biham, O., Liu, C., Shen L., \& Vidali, G. 1997b, Ap. J. 483, L131

Pirronello, V., Liu, C., Roser J.E., \& Vidali, G. 1999, A\&A A 344, 681

Roser, J.E., Manicó, G., Pirronello, V., \& Vidali, G. 2002, Ap. J. 581, 276

Roser, J.E., Swords S., \& Vidali, G. 2003, Ap. J. 596, L55

Sandford, S.A. \& Allamandolla, L.J. 1993, Ap. J. 409, L65

Shalabiea, O.M., Caselli, P., \& Herbst, E. 1998, Ap. J. 502, 652

Smoluchowski, R. 1981, Ap. Space Sci. 75, 353

Stantcheva, T., Caselli, P., \& Herbst, E. 2001, A\& A 375, 673

Stantcheva, T., Shematovich, V.I., \& Herbst, E., 2002, A\& A 291, 1069

Stantcheva, T. \& Herbst, E. 2003, MNRAS 340, 983

Takahashi, J., Masuda, K., \& Nagaoka, M. 1999, MNRAS 306, 22

Vidali, G., Roser, J., Manicó, G., Pirronello, V., Perets, H.B., \& Biham, O. 2005, Journal of Physics: Conference Series 6, 36

Vidali, G., Roser, J., Manicó, G., \& Pirronello, V. 2005, IAU Sympusium 231, this volume

Voshchinnikov, N.V., Semenov, D.A., \& Henning, T. 1999, A\&A A 349, L25

Weingartner, J.C. \& Draine, B.T. 2001, Ap. J. 548, 296

Williams, D.A. 1968, Ap. J. 151, 935

Williams, D.A. 1998, Faraday Discussions 109, 1 


\section{Discussion}

HORNEKÆR: Will the inclusion of chemisorption sites on dust grains turn even small interstellar grains into efficient contributors to $\mathrm{H}_{2}$ formation in photon dominated regions (PDRs) because thermal spikes could activate recombination of chemisorbed $\mathrm{H}$ atoms?

BiнAм: In our analysis so far we have not included fluctuations in grain temperatures. Such fluctuations are expected to be significant only for grains of radii smaller than about $10 \mathrm{~nm}$. Chemisorbed $\mathrm{H}$ atoms are expected to contribute to $\mathrm{H}_{2}$ formation when grain temperatures reach several hundred degrees $(\mathrm{K})$. However, a careful analysis is required in order to quantify the contribution of such mechanism.

AIKAWA: You concluded that $\mathrm{H}$ migrates by thermal hopping, while Cazaux fits the same experimental data assuming tunneling. Would you tell me why you need to exclude tunneling and why you and Cazaux reached different conclusions on H-migration?

BiнAm: The temperature programmed desorption (TPD) curves obtained for the polycrystalline olivine sample (Katz et al. 1999) exhibit second-order kinetics at lower coverage, namely, as the coverage decreases, the TPD peak shifts to higher temperatures. This indicates that the HD molecules are formed by thermal hopping during the TPD run. If tunneling alone provided sufficient mobility for $\mathrm{H}$ atoms to diffuse and recombine during irradiation, when the surface temperature is around $6 \mathrm{~K}$, all TPD curves would be of first-order kinetics. For other samples, such as amorphous carbon, the evidence for second order kinetics is not as strong and tunneling is not ruled out completely. 